\title{
Clival Chordoma With Brainstem Invasion
}

\author{
Amit R.L. Persad, Bradford Mechor, Yves Starreveld
}

Keywords: chordoma, clival chordoma, intradural, intra-axial

doi:10.1017/cjn.2017.279

Can J Neurol Sci. 2018; 45: 235-237

Chordoma is a rare neoplasm derived from remnants of the fetal notochord, with incidence of approximately 1/1,000,000. Classic locations for chordoma include the lumbosacral spine (30-50\%) and clivus (30-35\%). ${ }^{1}$ Clival chordoma has a high rate of recurrence due to its aggressive biology as well as technical difficulty with gross total resection. These tumors frequently present with neuro-ophthalmologic findings. ${ }^{2}$ Intradural and extradural extension of clival chordoma are known phenomena, but brainstem involvement is exceedingly rare.

A 62-year-old man presenting with right-sided cranial nerve VI palsy was found on MRI to have a large sellar mass with invasion of the retroclival space, thought to be an aggressive pituitary macroadenoma. The patient was booked for endoscopic transsphenoidal resection of this tumor. Intraoperatively, the tumor was noted to erode through the floor of the sella turcica and involved bony destruction of the clivus, consistent with chordoma. The tumor was noted to extend through a small dural defect into the prepontine cistern. Near gross total resection of the tumor was achieved, except a small portion anterior to the brainstem. Final pathology revealed chordoma. The patient had two repeat transsphenoidal resections 10 months and 18 months after his initial debulking, with intraoperative note made of brainstem invasion. He also received radiation therapy following his third surgery. He was then followed with MRI to monitor recurrence at 6-month intervals.

The patient remained free of recurrence until 1 year after his third operation. At that time radiologic recurrence was noted with only minor symptoms. After 6 months, the patient developed lower cranial nerve deficits including swallowing issues and drop attacks. An attempt at surgical decompression was undertaken via a retrosigmoidal approach, but the tumor had become too adherent to the brainstem, extending from the prepontine cistern into the belly of the pons with cystic component (Figure 1), with adjacent signal changes on FLAIR and T2 images. Diffusion weighted imaging showed some areas of restriction suggestive of infarction. The patient passed away during admission, likely from brainstem perforator infarct (Figures 2-4).

Brainstem invasion of clival chordoma is exceedingly rare, with only four cases reported in the literature. ${ }^{3-5}$ Aggressive extradural and intradural involvement is well known. Occasionally, chordoma can occur as a primary intradural lesion, which can be quite benign with good outcomes, though can rarely exhibit aggressive character. ${ }^{6}$ Other differential diagnosis for chordoma

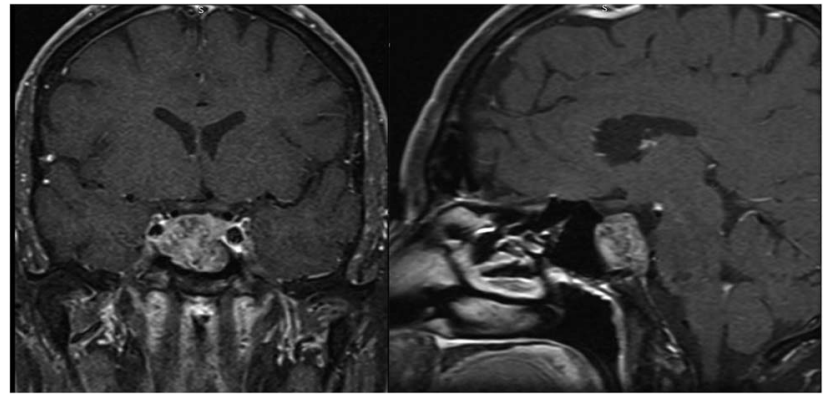

Figure 1: Coronal and sagittal post-gadolinium T1 magnetic resonance images showing clival chordoma at the time of diagnosis. The tumor demonstrates destruction of the clivus and involvement of the prepontine cistern but no intrapontine extension.

includes fetal notochord, ecchordosis physaliphora, atypical meningioma and chondrosarcoma. ${ }^{1}$

Treatment of chordoma is by surgical debulking. Conventional radiotherapy adds no benefit to treatment of these tumors, though postoperative proton therapy has been shown to improve survival. ${ }^{7}$ Surgical approaches include transsphenoidal, transcranial, transoropharygeal and maxillary osteotomy. ${ }^{7}$ The endoscopic transsphenoidal approach represents a useful, minimally invasive option with good visualization of the sella, clivus and retroclivus. $^{6,8,9}$ Patients with chordoma require extensive follow-up due to high risk for recurrence.

Clival chordoma is a rare intracranial neoplasm with many presentations. Extensive follow-up is required for patients with these tumors. Posterior fossa involvement and especially brainstem extension are associated with several catastrophic complications, including basilar artery compression and lower cranial nerve deficits.

From the Division of Neurosurgery, Department of Surgery, University of Saskatchewan, Saskatoon, SK, Canada (ARLP); Division of Otolaryngology-Head and Neck Surgery, Department of Surgery, University of Calgary, Calgary, AB, Canada (BM); Division of Neurosurgery, Department of Clinical Neurosciences, University of Calgary, Calgary, AB, Canada (YS).

Received June 27, 2017. Revised August 20, 2017. Date of Acceptance October $11,2017$.

Correspondence to: Dr. Yves Starreveld, Division of Neurosurgery, Department of Clinical Neurosciences, University of Calgary, Foothills Medical Centre, 140329 St NW, Calgary, AB, T2N 2T9, Canada. Email: starreveld.office@gmail.com 


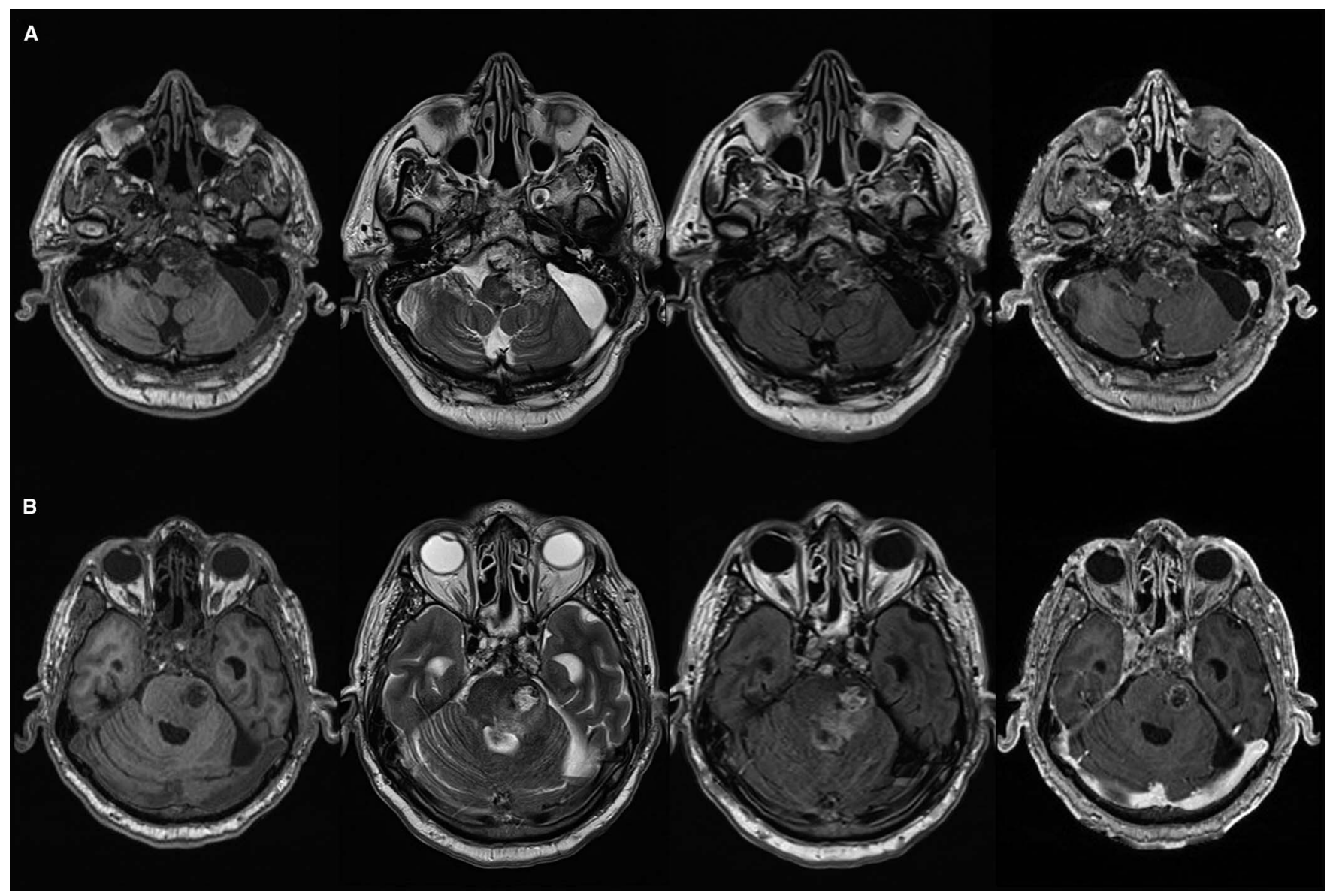

Figure 2: Axial T1, T2, FLAIR and post-gadolinium T1 magnetic resonance images showing clival chordoma and T2/FLAIR signal change in prepontine cistern $(A)$ with intrapontine invasion $(B)$.

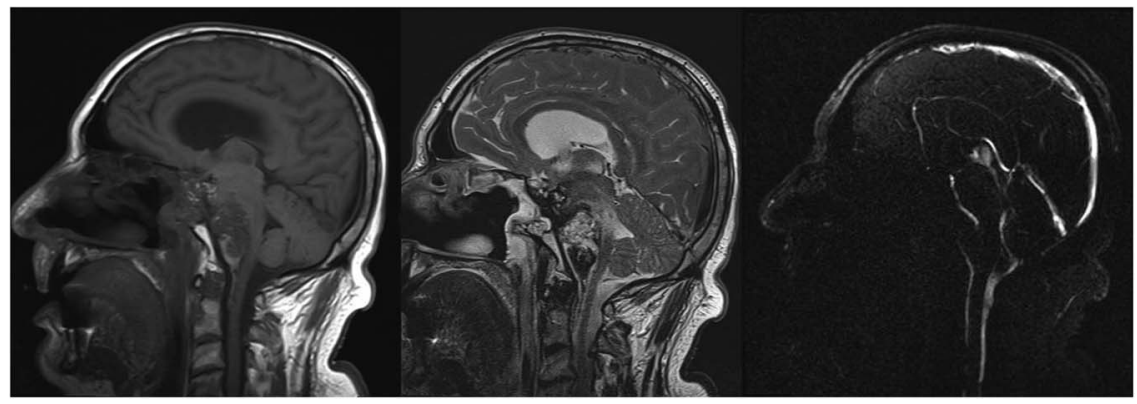

Figure 3: Sagittal T1, T2 and $P C$ in plane flow magnetic resonance images demonstrating pontine infiltration of the tumor and compression of the basilar artery.

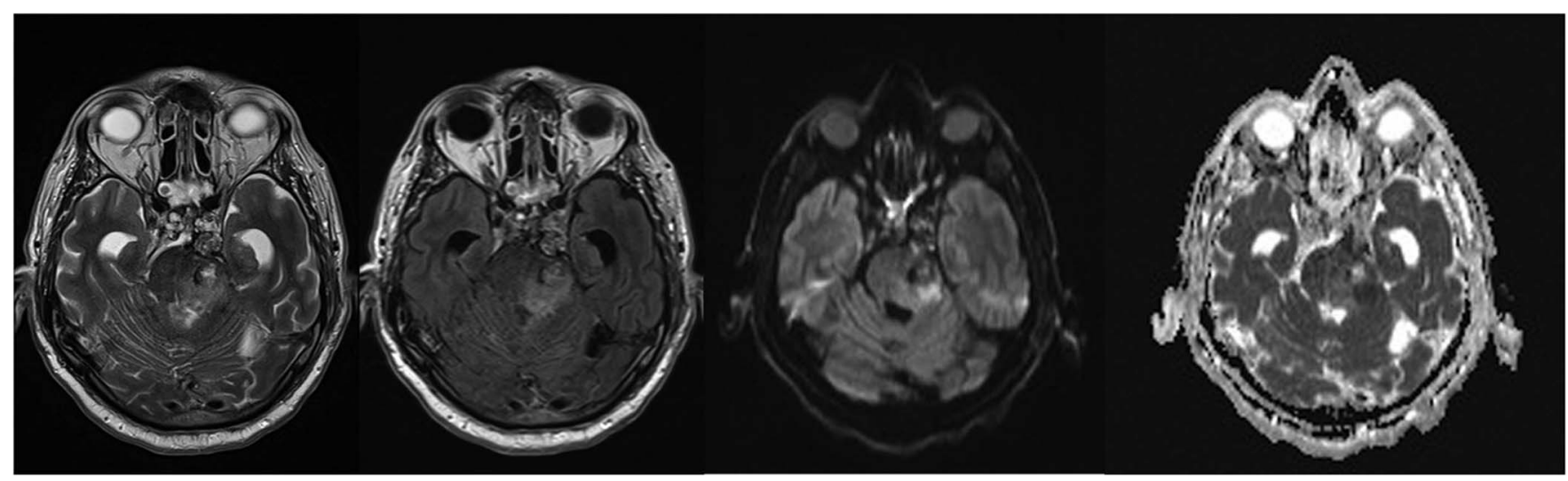

Figure 4: Axial T2, FLAIR, diffusion weighted imaging and ADC images showing areas of restricted diffusion within the brainstem. 


\section{DisClosure}

Amit R. L. Persad, Bradford Mechor and Yves Starreveld have no disclosures.

\section{REFERENCES}

1. Erdem E, Angtuaco EC, Van Hemert R, et-al. Comprehensive review of intracranial chordoma. Radiographics. 2003;23(4): 995-1009.

2. Michele SM, Samuel CL. Chordomas of the skull base: manifestations and management. Curr Opin Otolaryngol Head Neck Surg. 2003;11(5):324-7.

3. Herold C, Giordano M, Naka T, Gerganov V, Samii M, Samii A. Clivus chordoma in continuity with a large pontine cyst. Skull Base. 2009;19(2):177-81.
4. Lim GH. Clivus chordoma with unusual bone sclerosis and brainstem invasion. A case report with review of the radiology of cranial chordomas. Australas Radiol. 1975;19(3):242-50.

5. Zoli M, Milanese L, Bonfatti R, et al. Clival chordomas: considerations after 16 years of endoscopic endonasal surgery. J Neurosurg. 2017; $14: 1-10$.

6. Ito E, Saito K, Nagatani T, et al. Intradural cranial chordoma. World Neurosurg. 2010;73(3):194-7.

7. Colli B, Al-Mefty O. Chordomas of the craniocervical junction: followup review and prognostic factors. J Neurosurg. 2001;95:933-43.

8. Hong Jiang W, Ping Zhao S, Hai Xie Z, Zhang H, Zhang J, Yun Xiao J. Endoscopic resection of chordomas in different clival regions. Acta Otolaryngol. 2009;129:71-83.

9. Carrabba G, Dehdashti AR, Gentili F. Surgery for clival lesions: open resection versus the expanded endoscopic endonasal approach. Neurosurg Focus. 2008;25(6):E7. 